\title{
Pseudo-acylceramide with Linoleic Acid Produces Selective Recovery of Diminished Cutaneous Barrier Function in Essential Fatty Acid-deficient Rats and Has an Inhibitory Effect on Epidermal Hyperplasia
}

\author{
Genji Imokawa, Yukihiro Yada, Kazuhiko Higuchi, Minehiro Okuda, Yukihiro Ohashi, and Akira Kawamata \\ Tochigi Research Laboratories, Kao Corporation, Tochigi 321-34, Japan
}

\begin{abstract}
Pseudo-acylceramides with different acyl properties were investigated for their capacity to restore diminished barrier function in essential fatty acid-deficient rats. Daily topical applications of synthetic pseudo-acylceramides containing ester-linked linoleic acid caused a dose-dependent, significant reduction of transepidermal water loss (TEWL). Both other pseudo-acylceramides with ester-linked oleic acid or saturated alkyl chains and ordinary ceramides exhibited a poor effect on recovery of TEWL. Furthermore, pseudoceramide containing ether-linked linoleic acid, which is biologically inactive in terms of degradation by hydrolytic enzymes, also induced a significant and similar increase in the barrier function. This restoration of barrier function by pseudo-acylceramides with linoleic acid was accompanied by suppressed DNA synthesis in the EFAD rat epidermis. In UVB-irradiated guinea pig skin, topical applications of the pseudo-acylceramides with linoleic acid immediately after the exposure significantly reduced epidermal hyperplasia, secondary to markedly diminished barrier disruption, whereas linoleic acid itself did not. A comparison of both the anti-hyperplasia and the barrier recovery effects in the series of pseudo-ceramide derivatives examined revealed that the suppressive effect on the induced epidermal hyperplasia was paralleled by the recovery of the barrier defect in EFAD rats. These findings directly suggest that acylceramide with an ester-linked linoleic acid has an essential role in the epidermal permeability barrier. (J. Clin. Invest. 1994. 94:89-96.) Key words: permeability barrier • pseudo-acylceramide • epidermal hyperplasia • linoleic acid
\end{abstract}

\section{Introduction}

Sphingolipids are an important constituent of the permeability barrier of the stratum corneum (1). However, the predominant species of ceramides associated with this barrier function remain to be clarified. Recent studies of essential fatty acid deficiency

Address all correspondence to Genji Imokawa, Tochigi Research Laboratories, Kao Corporation, 2606, Akabane, Ichikaimachi, Haga, Tochigi, 321-34, Japan.

Received for publication 7 August 1993 and in revised form 2 February 1994.

\section{J. Clin. Invest.}

(c) The American Society for Clinical Investigation, Inc.

0021-9738/94/07/0089/08 \$2.00

Volume 94 , July $1994,89-96$
(EFAD $)^{1}$ in animals have shown that acylceramide with linoleic acid in an ester linkage is replaced by acylceramide with oleic acid in parallel with the progression of barrier defects that occur when a linoleic acid-depleted diet is given to animals $(2,3)$, suggesting the importance of the former in barrier function. However, there is no direct evidence to show whether such acylceramides are involved in barrier functions. During studies on the water-retaining mechanism of the stratum corneum (47 ), we established pseudo-ceramide structures that function in a manner similar to that of naturally occurring ceramides (8, 9). In an attempt to directly study the role of acylceramide with linoleic acid in controlling barrier function, we synthesized pseudo-acylceramides with different acyl properties and examined their potential in recovering diminished barrier function in EFAD rats. Based upon recent evidence $(10,11)$ of the relevance of perturbed barrier function to epidermal proliferation, we also assessed the effects of topical applications of these agents on the epidermal hyperplasia that occurs in barrier-interrupted guinea pig skin.

\section{Methods}

EFAD animals. Wistar rats (male, 4-wk old) received a diet consisting of casein, sucrose, choline, a mixture of salts, the fat-soluble vitamins, $\mathrm{A}, \mathrm{D}$, and $\mathrm{E}$, and the water-soluble B vitamins, to which $5 \%$ corn oil and inositol $(1 \mathrm{mg} / \mathrm{g})$ was added. The deficient group were fed identical quantities of all of the above for 8-10 wk, except that equal parts of hydrogenated coconut oil $\left(C_{8: 0}, 6.8 ; C_{10: 0}, 5.7 ; C_{12: 0}, 45.1 ; C_{14: 0}, 19.1\right.$; $\mathrm{C}_{16: 0}, 10.3 ; \mathrm{C}_{18: 0}, 11.8 ; \mathrm{C}_{18: 2}, 0.3 ; \mathrm{C}_{20: 0}, 0.1$ weight \%) were substituted for corn oil.

Materials. Pseudo-ceramide derivatives were synthesized essentially according to the method described previously (9). The chemical structures of the synthesized compounds and their assigned compound numbers are shown in Fig. 1. We purchased $\left[{ }^{14} \mathrm{C}\right]$ linoleic acid $(60 \mathrm{mCi} /$ $\mathrm{mmol}$ ) from New England Nuclear (Boston, MA) and synthesized pseudo-acylceramide containing ester-linked $\left[{ }^{14} \mathrm{C}\right]$ linoleic acid([linoleoyl$1-{ }^{14} \mathrm{C}$ ]BRS-001, $43 \mathrm{mCi} / \mathrm{mmol}$ ).

The synthetic compounds were emulsified at concentrations of $1-$ $10 \%$, in squalane containing $1 \% \alpha$-monomethyl heptadecyl glyceryl ether (GE). This emulsion ( $18 \mu \mathrm{g} / 4 \times 8 \mathrm{~cm}^{2}$ ) was applied once a day for 2-3 wk to shaved skin. EFAD controls and normal controls were treated with vehicle only (squalane containing $1 \% \mathrm{GE}$ ).

Transepidermal Water Loss Measurement. Transepidermal water loss (TEWL) was measured at $22-24^{\circ} \mathrm{C}$ and $55 \%$ humidity with an Evaporimeter EP1 apparatus (SERVO MED Co. Ltd.) $2 \mathrm{~h}$ after rinsing the applied skin area with warm water.

Human keratinocyte culture. Human keratinocytes, purchased from Sunko-junyaku Co. Ltd. (Epipak) were cultured on collagen-coated

1. Abbreviations used in this paper: EFAD, essential fatty acid deficiency; GE, $\alpha$-monomethy heptadecyl glyceryl ether; TEWL, transepidermal water loss. 


\begin{tabular}{|c|c|}
\hline $\begin{array}{l}\text { COMPOUND } \\
\text { NUMBER }\end{array}$ & CHEMICAL STRUCTURE \\
\hline BRS-000 & ำmำ \\
\hline BRS-001 & 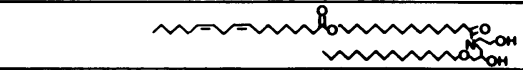 \\
\hline BRS-002 & mกn工m \\
\hline BRS-003 & nomm nom \\
\hline BRS-004 & minm \\
\hline BRS-005 & m=ก-กำกำ \\
\hline BRS-006 & 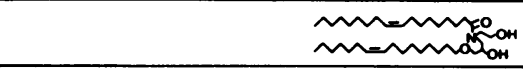 \\
\hline BRS-007 & 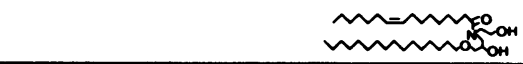 \\
\hline BRS-008 & mำ \\
\hline BRS-009 & mmm \\
\hline BRS-010 & movannommenom \\
\hline BRS-011 & mmmmm \\
\hline BRS-012 & 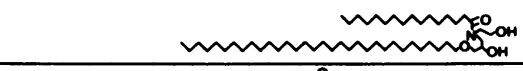 \\
\hline BRS-013 & Wn=mionm \\
\hline BRS-014 & 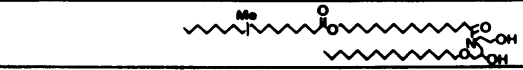 \\
\hline BRS-015 & ñV-nnionnm \\
\hline BRS-016 & nranmionm nom \\
\hline BRS-017 & m=rem \\
\hline BRS-018 & monnionm \\
\hline BRS-019 & mixn \\
\hline
\end{tabular}

Figure 1. Synthetic pseudo-ceramides and their chemical structures.

Petri dishes in MCDB-153 medium, supplemented with $200 \mathrm{U} / \mathrm{ml}$ penicillin, $100 \mu \mathrm{g} / \mathrm{ml}$ streptomycin, $0.63 \mu \mathrm{g} / \mathrm{ml}$ fungizone (Flow Laboratories, Inc., McLean, VA), $70 \mu \mathrm{g} / \mathrm{ml}$ bovine pituitary extract (PelFreeze Biologicals, Rogers, AR), $1 \mathrm{ng} / \mathrm{ml}$ epidermal growth factor (Sigma Chemical Co., St. Louis, MO), $50 \mu \mathrm{M}$ hydrocortisone (GIBCO BRL, Gaithersburg, MD), $0.1 \mathrm{mM}$ ethanolamine, and $0.1 \mathrm{mM}$ phosphoethanolamine (Sigma Chemical Co.).

DNA synthesis. DNA synthesis in the epidermis was evaluated by measuring the incorporation of methyl- $\left[{ }^{3} \mathrm{H}\right]$ thymidine into tissue in an skin organ culture, as described elsewhere (12). $1 \mu \mathrm{Ci} / \mathrm{ml}$ of methyl$\left[{ }^{3} \mathrm{H}\right]$ thymidine was incubated for $4 \mathrm{~h}$ in the skin organ culture, which was biopsied immediately after the topical application of the synthetic compounds ceased. Organ cultured skin was separated by dispase digestion into epidermis and dermis. The radioactivity incorporated into the epidermal sheet $\left(5 \times 5 \mathrm{~mm}^{2}\right)$ after $5 \%$ TCA treatment was then measured using a liquid scintillation counter. The DNA synthesis of human keratinocytes was measured by determining methyl- $\left[{ }^{3} \mathrm{H}\right]$ thymidine incorporation in cultured cells. In proliferative stages conditioned by the above described medium, the cells were pulse-labeled with $1 \mu \mathrm{Ci}$ of
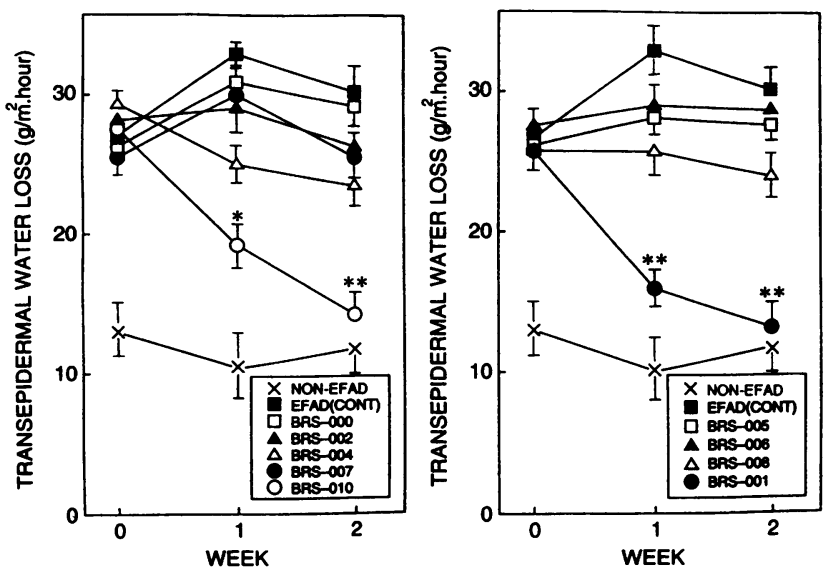

Figure 2. Time course study of TEWL changes induced by application of several pseudo-ceramides at a concentration of $10 \%$. In non-EFAD $(x)$ and EFAD (CONT) $(\square)$ experiments, animals were treated with vehicle (squalane containing $1 \% \mathrm{GE}$ ) only. $N=3 .^{*}, P<0.05 ; * *, P$ $<0.01$ as compared to vehicle-treated control (EFAD [CONT]).

methyl- $\left[{ }^{3} \mathrm{H}\right]$ thymidine $(25 \mu \mathrm{Ci} / \mathrm{mmol}$ ) for the last $4 \mathrm{~h}$ of a $24-\mathrm{h}$ incubation and the incorporated labeled thymidine was determined using a liquid scintillation counter, after the cells were washed twice with cold Hank's balanced solution, followed by 5\% TCA treatment and solubilization with $1 \mathrm{~N} \mathrm{NaOH}$.

Assay of degradative properties. $20 \mu \mathrm{l}$ of $\left[{ }^{14} \mathrm{C}\right] \mathrm{BRS}-001\left(10^{5}\right.$ $\mathrm{dpm}$ ) in chloroform was applied to the EFAD rat shaved skin using a penetration chamber. After $24 \mathrm{~h}$, the skin was washed with chloroform for a few seconds, separated, and stripped with cyanoacrylate resin and scotch tape to obtain whole layers of the stratum corneum. Lipids in the stripped stratum corneum were extracted with $20 \mathrm{ml}$ of chloroform. The extracts were solubilized in $100 \mu \mathrm{l}$ chloroform and analyzed by HP-TLC. HP-TLC plates were developed with chloroform/methanol (10:1) to resolve pseudo-acylceramide and linoleic acid. After development, the chromatograms were air-dried and scanned for radioactivity (System 2001 imaging scanner; Biosan Inc., Wash. DC). The remnant, which consists of epidermis and dermis, was also extracted with chloroform for HP-TLC analysis. In a separate experiment, the remnant was solubilized in $2 \mathrm{~N} \mathrm{NaOH}$ and neutralized by $2 \mathrm{~N} \mathrm{HCl}$ for scintillation counting.

Epidermal hyperplasia. The effects of the agents on epidermal hyperplasia were evaluated by measuring the thickness of the epidermis, excluding the stratum corneum, in guinea pig ear skin. The synthetic compounds, emulsified at a concentration of $10 \%$ in squalane, were applied twice a day for $1 \mathrm{wk}$ to guinea pig ear skin immediately after the skin had been irradiated at 2 MED dose of UVB light (Toshiba FL20E lamp) with an intensity of $0.6 \mathrm{~mW} / \mathrm{cm}^{2}$ at $305 \mathrm{~nm}$ (1 MED $=0.29 \mathrm{~J} / \mathrm{cm}^{2}$ ) or immediately after it had been stripped 15 times with scotch tape. Thereafter, the skin was biopsied and stained with hematoxylin and eosin to measure the epidermal thickness. The thickness of the Malpighian layer of the epidermis, namely the distance from the dermalepidermal interface to the junction between the granular and keratin layers, was measured microscopically in 200 sites in the section using a calibrated eyepiece oriented perpendicular to the dermal-epidermal interface.

\section{Results}

Effects of pseudo-acylceramides on impaired barrier function. Fig. 2 ( $A$ and $B$ ) shows the time course of TEWL changes in relation to the application of several pseudo-ceramides at a 


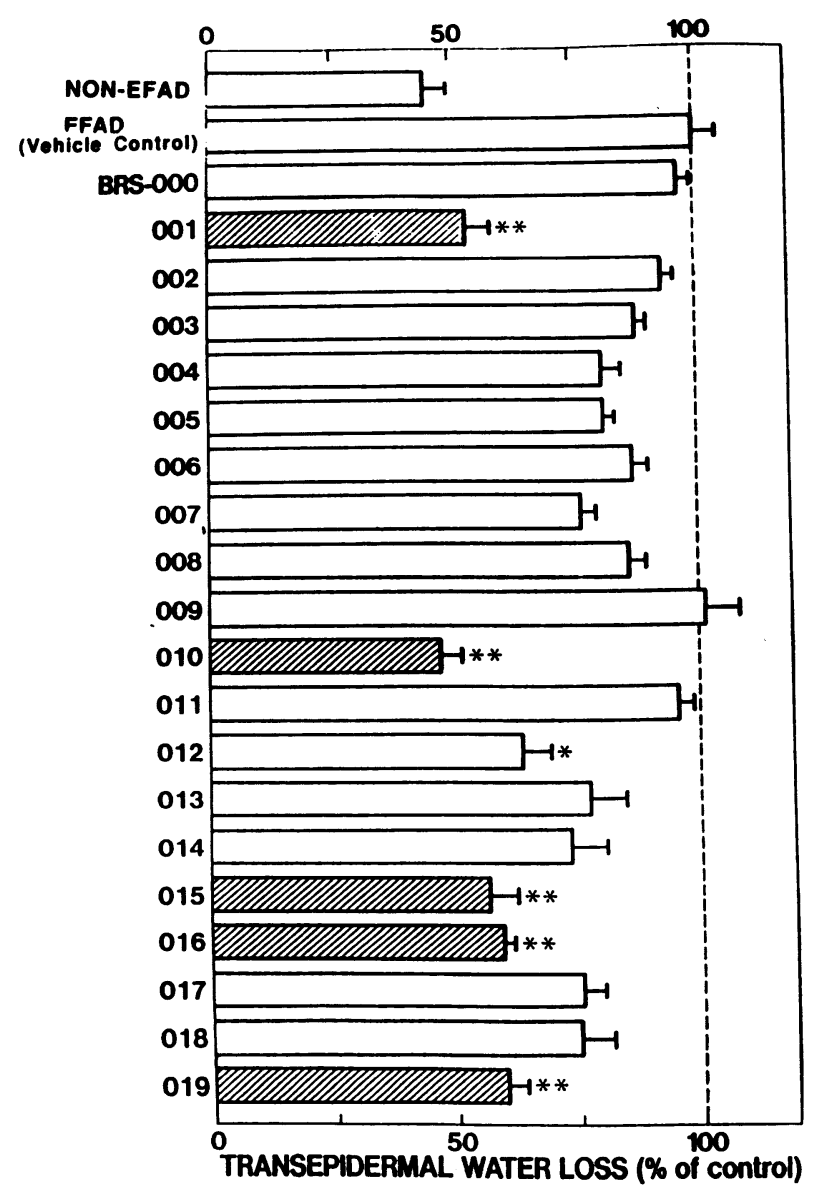

Figure 3. The effects of topical application (10\%) of several types of pseudo-ceramides on barrier function as measured by TEWL changes (\% of control) after $14 \mathrm{~d}$ of treatment. Each sample treatment value is expressed as a percentage of the control relative to the value of EFAD (vehicle control). $N=3 .{ }^{*}, P<0.05 ;{ }^{* *}, P<0.01$.

concentration of $10 \%$. Topical application of pseudo-acylceramide with ester-linked linoleic acid (BRS-001) produced a marked reduction in TEWL after 2 wk of treatment, whereas no significant reduction was associated with other pseudoceramides (BRS-000, 004), including those with unsaturated alkyl chains (BRS-005, 006, 007, and 008) or with long alkyl chains (BRS-002). To rule out the possibility that the applied pseudoacylceramides were hydrolyzed by epidermal hydrolytic enzymes to yield linoleic acid, which can evoke barrier recovery, we studied the barrier recovery effects of a pseudo-ceramide (BRS-010) in which the ester linkage was replaced by an ether bond; this agent contained ether-linked linoleic acid. As seen in Fig. 2 B, BRS-010 also significantly corrected barrier disruption in the EFAD rat. Fig. 3 summarizes the effect of the topical application ( $10 \%$ ) of several types of pseudo-ceramides on barrier function, as measured by TEWL after 2 wk of treatment. Pseudo-acylceramides with linoleic acid in an ester linkage (BRS-001, 015, and 016) and the pseudo-ceramide containing ether-linked linoleic acid (BRS-010) produced a marked recovery in TEWL, up to control level, whereas the pseudo-acylceramide with ester-linked oleic acid (BRS-013) had poor effects on recovery. In contrast to the marked recovery effect produced by BRS-001, a different pseudo-acylceramide containing ester-

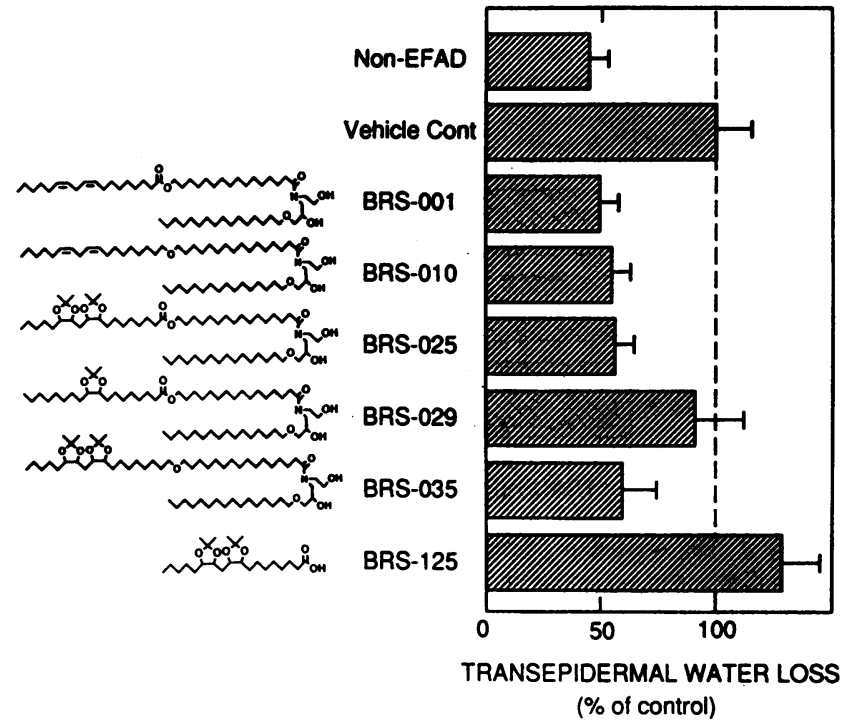

Figure 4. The effects of topical application (10\%) of the pseudo-acylceramide with dioxsoran-adduct acid on barrier function as measured by TEWL changes (\% of control) after $14 \mathrm{~d}$ of treatment. Each sample treatment value is expressed as a percentage of control relative to the value of EFAD (vehicle cont). $N=3 .^{*}, P<0.05{ }^{* *}, P<0.01$.

linked linoleic acids and an amide-linked odd-numbered fatty acid (BRS-017) (as compared with the amide-linked even numbered fatty acid in BRS-001), produced no significant recovery of TEWL. Further, ordinary pseudo-ceramides, even with linoleic acid (BRS-005), also produced no significant recovery effects. Of considerably importance for excluding the possible action by decomposed linoleic acid from acylceramides is the fact that, as shown in Fig. 4, the pseudoceramides with ester or ether-linked dioxsoran adduct-acid derivatives (BRS-025 and 035 ) in which two double bonds in linoleic acid were replaced with dioxsoran, also induced a similar and significant recovery of TEWL despite the fact that there was no significant recovery by the possible released acid, namely the dioxsoran-adduct acid (BRS-125) as well as by the other pseudoceramide containing an ester-linked dioxsoran-adduct (BRS-029) in which double bond of oleic acid was replaced by dioxsoran. Fig. 5 shows the dose-dependent recovery of barrier function following the application of pseudo-acylceramide with linoleic acid in an ester linkage (BRS-001), as revealed by the reduction in TEWL.

Quantitation of the degradative properties of pseudo-acylceramide. To directly preclude the possibility that linoleic acid, which is generated by hydrolysis of BRS- 001 induced the barrier recovery, we determined whether linoleic acid emerges after a 24-h application of $\left[{ }^{14} \mathrm{C}\right]$ linoleic acid bearing BRS-001 to EFAD rat skin. When the tissue distribution of $\left[{ }^{14} \mathrm{C}\right] \mathrm{BRS}-001$ and $\left[{ }^{14} \mathrm{C}\right]$ linoleic acid released were examined after a $24 \mathrm{~h}$ application to EFAD rat skin, the radioactivity of the applied $\left[{ }^{14} \mathrm{C}\right] \mathrm{BRS}-001$ was confined to the stratum corneum without releasing $\left[{ }^{14} \mathrm{C}\right]$ linoleic acid (Fig. 6, Table I). Other tissue excluding stratum corneum (consisting mainly of epidermis and dermis) did not contain any radiolabeled materials, indicating the specific distribution of applied BRS-001 within the stratum corneum, lacking liberated linoleic acid. 


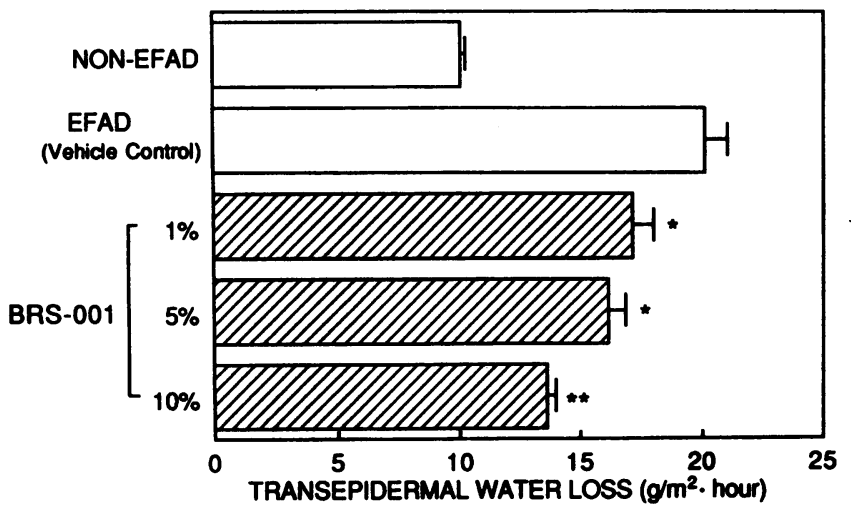

Figure 5. The dose-dependent recovery of barrier function following the application of pseudo-acylceramide with linoleic acid in ester linkage $(B R S-001)$ as shown by the reduced TEWL after 2 weeks of treatment. $N=3$. Bar indicates standard deviation from three experiments. ${ }^{*}, P$ $<0.05 ; * *, P<0.01$.

Effects of pseudo-acylceramides on epidermal DNA synthesis of EFAD rat. As shown in Fig. 7, in accordance with the observed recovery effect on barrier function, a 2-wk application of pseudo-acylceramide with ester-linked linoleic acid (BRS-001) and ether-linked pseudo-ceramide with linoleic acid (BRS-010) produced a significant reduction in DNA synthesis in the EFAD rat epidermis when measured in organ cultured skin, as compared with vehicle-treated controls, whereas a pseudo-acylceramide with oleic acid (BRS-013), and a saturated, branched alkyl chain pseudoacyceramide (BRS-014) and an ordinary pseudo-ceramide with long alkyl chains (BRS-011) had no significant inhibitory effect on DNA synthesis.

Effects of pseudo-acylceramides on UVB exposed or stripped skin of guinea pig. Consistent with the effect on EFAD rat skin, when $10 \%$ BRS-001 was applied daily for 1 wk immediately after exposing the ear skin of guinea pig to UVB irradiation at a dose of $2 \mathrm{MED}$, the induced disruption of barrier function as revealed by the increased TEWL value was signifi-
Table I. The Distribution of Topically Applied BRS-001 with Radiolabeled Linoleic Acid in the EFAD Rat Skin and Determination of Linoleic Acid Release

\begin{tabular}{lcc}
\hline & $\begin{array}{c}\text { Purity of } \\
\text { radiolabeled } \\
\text { BRS-001 }\end{array}$ & $\begin{array}{c}\text { Released } \\
\text { radiolabeled } \\
\text { linoleic acid }\end{array}$ \\
\hline $\begin{array}{l}\text { Before application } \\
\text { Stratum corneum after 24 h of } \\
\text { application }\end{array}$ & 97.0 & $\%$ \\
$\begin{array}{l}\text { Epidermis + dermis after 24 h } \\
\text { of application }\end{array}$ & 95.2 & N.D. \\
\hline
\end{tabular}

$\mathrm{ND}$, not detectable; the total radio-activity of the remnant (epidermis + dermis) was negligible. The purity of BRS-001 and the linoleic acid release was assessed by HPTLC of the chloroform extract from the stratum corneum or the remnant (epidermis +dermis) of skin to which BRS-001 was applied for $24 \mathrm{~h}$. The radioactivity in the remnant was also measured using a scintilation counter. The total radioactivity applied to HPTLC was $\sim 5,000$ and 4,800 dpm for the original sample before, and the stratum corneum extract after application, respectively.

cantly diminished (Fig. 8). To examine the inhibitory effect of BRS-001 and BRS-010 on the induced epidermal hyperplasia, BRS derivatives were applied daily to UVB-irradiated or tapestripped guinea pig skin; after $1 \mathrm{wk}$, the thickness of the epidermis was measured (Fig. 9). BRS-001 and BRS-010 produced a significant suppression in epidermal thickness as compared with vehicle-treated controls, whereas linoleic acid itself and ordinary pseudo-ceramides (BRS-000 and 011) did not (Fig. $10, A$ and $B$ ). It should be noted that there was no inflammatory reaction due to the applied materials including linoleic acid, during the experimental period.

Relationship between anti-hyperplasia and barrier recovery effect. Comparison of anti-hyperplasia and barrier recovery effects of this series of pseudo-ceramide derivatives (Fig. 11) revealed that the suppressive effect on the UVB-induced epider-

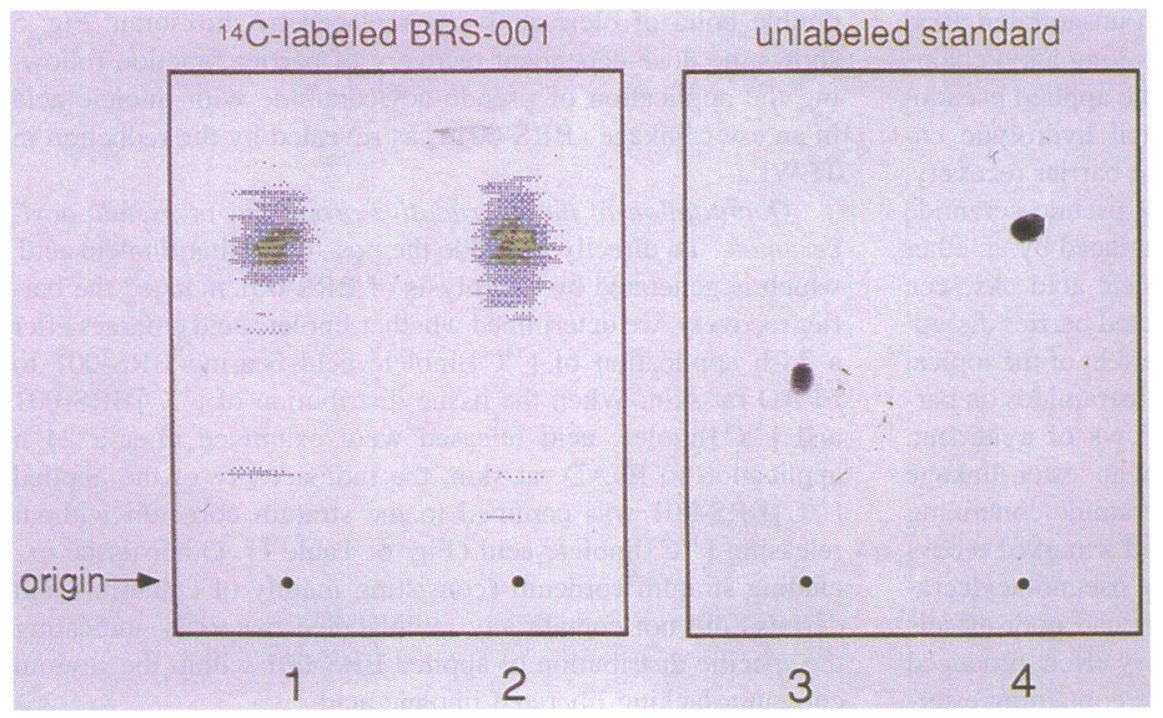

Figure 6. High Performance TLC of the extracts from stratum corneum of EFAD rat skin at 24 $\mathrm{h}$ after the application of ${ }^{14} \mathrm{C}-\mathrm{BRS}-001.20 \mu \mathrm{l}$ of $\left[{ }^{14} \mathrm{C}\right]$ BRS-001 $(43 \mathrm{mCi} / \mathrm{mmol})(100,000$ dpm) solubilized in chloroform was applied to EFAD rat skin. HP-TLC was developed with chloroform/methanol (10:1) and the chromatogram was scanned for radioactivity (BIOSCAN) in experiments 1 and 2, or in experiments 3 and 4 , sprayed with $10 \% \mathrm{CuSO}_{3}, 8 \% \mathrm{H}_{3} \mathrm{PO}_{4}$ aqueous solution, followed by charring on a $180^{\circ} \mathrm{C}$ hot plate. (1) Before application; (2) after $24 \mathrm{~h}$ of application; (3) unlabeled linoleic acid standard; and (4) unlabeled BRS-001 standard. 


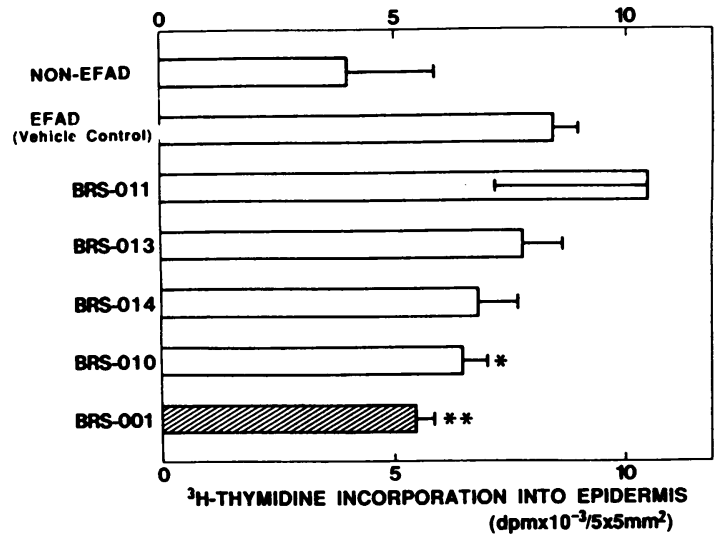

Figure 7. The effects of a 2-wk application of various pseudo-acylceramides on DNA synthesis in EFAD rat epidermis. Epidermal DNA synthesis was assessed in a 24-h organ culture of treated skin by measuring methyl- $\left[{ }^{3} \mathrm{H}\right]$ thymidine incorporation into the epidermis. $N=3$. Bar indicates standard deviation from three experiments. ${ }^{*}$, $P<0.01$.

mal hyperplasia was paralleled by the recovery of barrier defects in the EFAD rats. A different type of pseudo-acylceramide containing ester-linked linoleic acid (BRS-017), BRS-009, BRS000 , and BRS-011 produced neither recovery of TEWL nor a suppressive effect on epidermal hyperplasia, whereas BRS-001 and BRS-010 elicited both a significant recovery and suppression.

Effects of pseudo-acylceramide on DNA synthesis of cultured human keratinocytes. To rule out the possibility that BRS-001 can directly suppress DNA synthesis, which would have resulted in the observed inhibition of epidermal hyperplasia, we evaluated its inhibitory effect on DNA synthesis in human keratinocyte culture. We found that at concentrations of $0.0001-0.01 \%$ for $24 \mathrm{~h}, \mathrm{BRS}-001$ produced no inhibitory effect on thymidine uptake by human keratinocytes (data not shown).

\section{Discussion}

A large body of evidence supports the concept that stratum corneum lipids are a major determinant in mediating the epider-

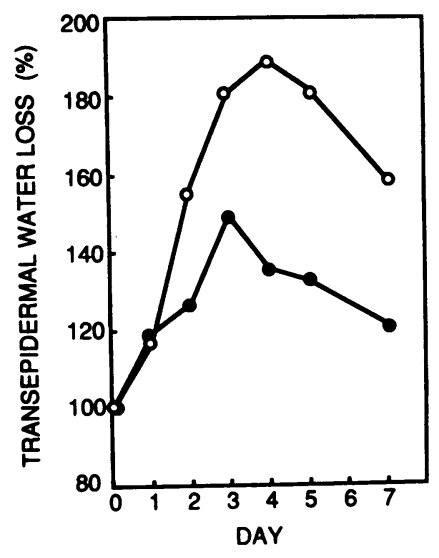

Figure 8. The effects of the application of pseudo-acylceramide with linoleic acid (BRS001 ) on impaired barrier function induced by UVB irradiation at a dose of 2 MED, as shown by TEWL changes. BRS-001 was applied daily at a concentration of $10 \%$ for 1 week to the UVB-exposed ear skin of a guinea pig. ( $O$ ) Vehicle (squalane solution containing $1 \%$ GE); (•) $10 \%$ BRS-001 in vehicle. mal permeability barrier $(13,14)$. Sphingolipid extraction and the subsequent barrier disruption that occurs following sequential treatment with acetone or petroleum ether have shown that sphingolipids are the principal mediators of barrier function (15). Sphingolipids, of which ceramides are the major constituents, are unique heterogeneous compounds including ceramides with different alkyl chain properties, an ester-linked fatty acid, and phytosphingosine bases. Among these, acylceramide with ester-linked linoleic acid is thought to be of major significance for the physical structure and function of the epidermal permeability barrier, through the assembly and organization of the extracellular lipid domains of the stratum corneum $(2,3)$. This hypothesis is principally based on the finding that the skin of essential fatty acid-deficient animals contains more acylceramide with predominantly oleic acid that has been substituted for linoleic acid, although there is no impairment in the formation of lamellar granules and the intercellular lipid sheet in the stratum corneum (3). However, the mode of action of acylceramide that contains ester-linked linoleic acid in the quisition of barrier function in the stratum corneum is $\mathrm{pc}$ understood. This is due mainly to this acylceramide bei
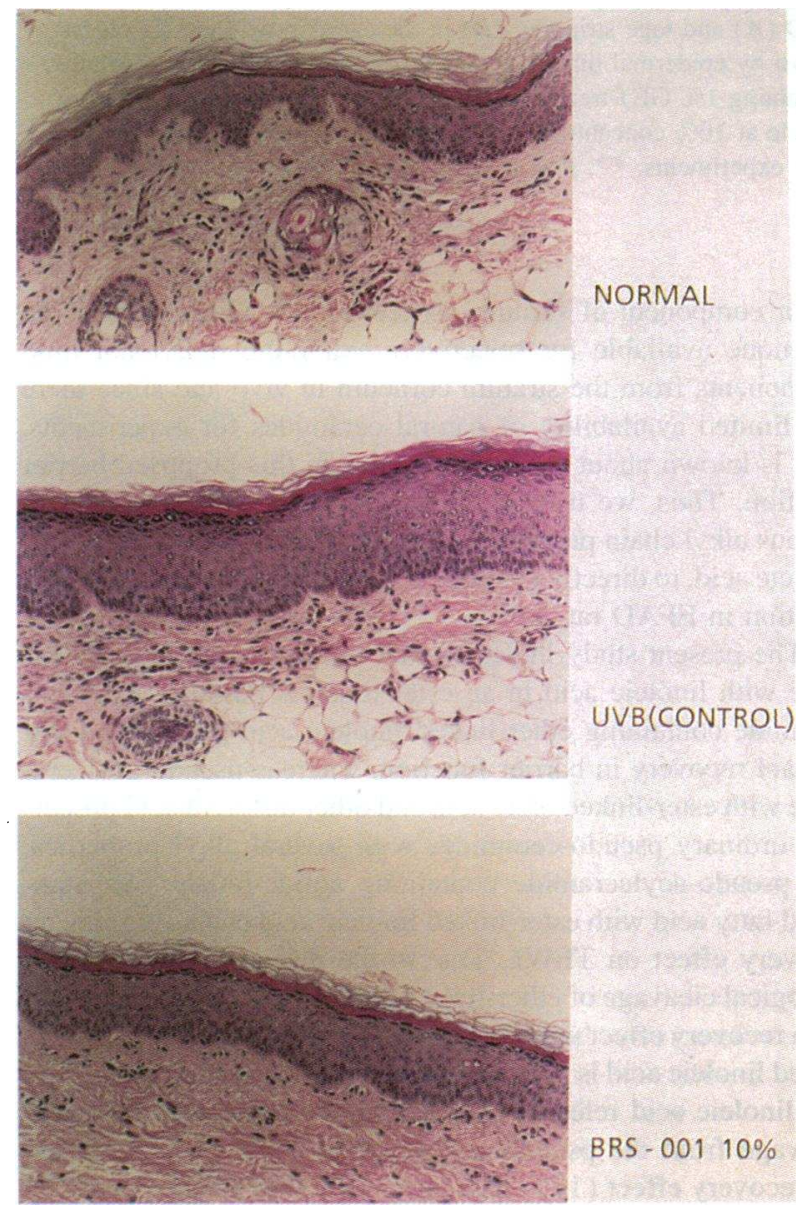

Figure 9. The inhibitory effect of pseudo-acylceramide with linoleic acid (BRS-001) on hyperplasia induced by UVB exposure in the ear skin of a guinea pig; hematoxylin and eosin stained skin specimens. (a) Before UVB exposure; (b) vehicle treatment after UVB exposure; and $(c) 1$ wk of treatment with samples at a concentration of $10 \%$ after UVB exposure. 


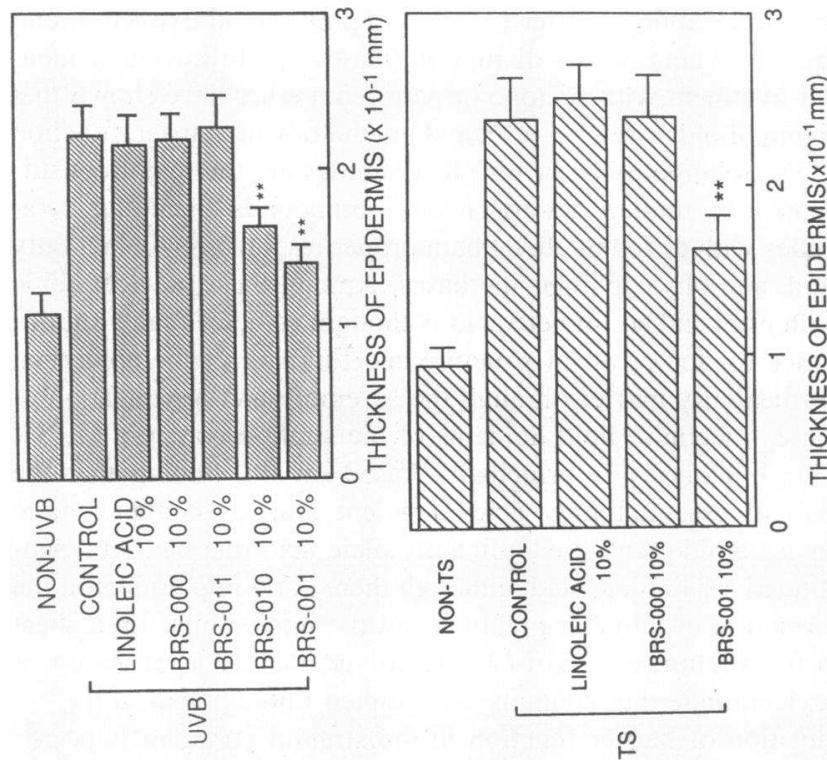

Figure 10. Inhibitory effects of pseudo-acylceramide with linoleic acid (BRS-001) and pseudo-ceramide containing ether-linked linoleic acid (BRS-010) on hyperplasia induced by UVB exposure at a dose of 2 $\operatorname{MED}(A)$ and tape stripping $(B)$ in the ear skin of a guinea pig, as shown by epidermal thickness. (Control) Vehicle (squalane solution containing $1 \% \mathrm{GE}$ )-treated control. All samples were emulsified in vehicle at $10 \%$ concentration. Bar indicates standard deviation from three experiments. $* *, P<0.01$.

minor component of stratum corneum lipids. Since there is no technique available for selectively extracting individual lipid components from the stratum corneum in vivo and since there is a limited availability of natural ceramides for experiments, little is known about their significance in this proposed barrier function. Thus, we used synthetic pseudo-acylceramides with various alkyl chain properties, including those with ester-linked linoleic acid, to directly evaluate their capacity to recover barrier function in EFAD rat skin.

The present study indicated that not only pseudo-acylceramide with linoleic acid in an ester linkage, but also a pseudoceramide containing ether-linked linoleic acid produced a significant recovery in barrier function, whereas pseudo-acylceramide with ester-linked oleic acid and other different acyl properties, ordinary pseudo-ceramides with several alkyl properties, and pseudo-acylceramide containing amide-linked odd numbered fatty acid with ester-linked linoleic acid had no significant recovery effect on TEWL. Due to the difficulty of producing biological cleavage of ether-linked linoleic acid, evidence showing a recovery effect with the pseudo-ceramide containing etherlinked linoleic acid is very important in ruling out the possibility that linoleic acid released through enzymatic or nonenzymatic cleavage from the pseudo-acylceramide could have produced the recovery effect (16). This possibility was also excluded by our experiments using pseudo-acylceramide with radiolabeled linoleic acid; all the radioactivity of $\left[{ }^{14} \mathrm{C}\right] \mathrm{BRS}-001$ was confined to the stratum corneum without releasing $\left[{ }^{14} \mathrm{C}\right]$ linoleic acid after a 24-h application to EFAD rat skin. Furthermore, the pseudo-acylceramide containing ester-linked linoleic acid and amide-linked odd numbered fatty acid (as compared with

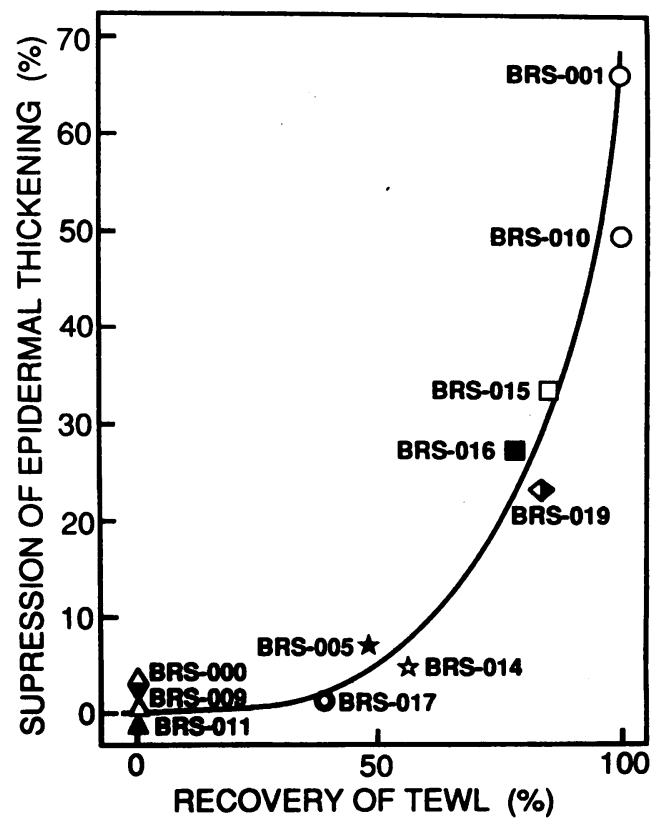

Figure 11. A comparison of anti-hyperplasia and barrier recovery effects obtained with a series of pseudo-ceramide derivatives. Anti-hyperplasia was evaluated by measuring epidermal thickness after 1 wk of treatment with samples at a concentration of $10 \%$, on UVB-exposed guinea pig ear skin. Thickness is expressed as a percentage relative to UVB-exposed, vehicle-treated controls. The barrier recovery effect was evaluated by measuring TEWL after a 2-wk treatment with samples at a concentration of $10 \%$, in EFAD rats; values are expressed as a percentage recovery of TEWL by assigning recovery to non-EFAD level as $100 \%$.

the amide-linked even-numbered fatty acid in BRS-001) which is presumed to have a similar likelihood of being degraded by hydrolytic processes, was not effective in recovering TEWL. The above findings indicate that the acylceramide with esterlinked linoleic acid plays an important role in cutaneous barrier function.

In EFAD animals, linoleic acid is an essential requirement for maintaining the epidermal barrier function. However, the recent discovery of linoleate-rich acylceramides (17) has suggested mechanisms whereby the nature of the ester-linked linoleic acid is associated directly with the formation of the intercellular lamellae and the stabilization of an orderly and impermeable physical structure in the stratum corneum (3). Thus, the beneficial effects of topically applied linoleic acid on EFAD have been ascribed to their conversion to linoleaterich acylceramides rather than to the cyclooxygenase or lipoxygenase products (18). Columbinic acid also corrects the barrier-deficiency state despite the failure of the cyclooxygenase or lipoxygenase products to normalize the epidermal water loss $(19,20)$. This also provides evidence that the nature of the ester-linked fatty acid in the acylceramides regulates the permeability barrier, although the recovery effect of the acylceramide with ester-linked columbinic acid remains to be clarified. The evidence for the structural significance of acylceramide with linoleic acid in the barrier function is also supported by our observation that, whereas the pseudoacylceramides with dioxsoran-adduct acid (BRS-025, 035) 
in which the double bonds of linoleic acid are replaced by dioxsoran also have a significant potential of recovering barrier function in the EFAD rat despite the failure of its possible degradation compound, dioxsoran-adduct acid (BRS-125), to correct barrier function, the other pseudo-ceramide containing ester-linked dioxsoran-adduct (BRS-029) in which the double bond of oleic acid is replaced by dioxsoran is not at all effective. Based upon the speculation $(18,21)$ that the $O$-polyoxyacylceramide generated via a specific lipoxygenase is relevant to the maintenance of epidermal barrier function, the structural features of ester- or ether-linked dioxsoran-adduct acid for acquiring barrier restoration may account for the significance of the oxygenated polyunsaturated fatty acid in the barrier function.

Several lines of evidence have demonstrated that perturbations in barrier function stimulate epidermal lipid synthesis through increased water transit in the epidermis (22). Furthermore, as an extension of the role of barrier function, modification of barrier function by solvents or by occlusion of skin with impermeable wraps, without causing nonspecific injury to the epidermis, has a great influence on epidermal DNA synthesis (10). Recent evidence also indicated that barrier disruption stimulates cytokine production which can modulate lipid and DNA synthesis in epidermis (23). Therefore, it is of considerable interest to determine whether barrier enhancing substances can suppress the epidermal hyperplasia induced by stimuli which interrupt barrier function. If this were so, it would provide strong evidence for the important role of acylceramide with linoleic acid in barrier function. In accordance with this relationship between DNA synthesis and barrier function, we found that topical application of the most active substance, pseudo-acylceramide with esterlinked linoleic acid (BRS-001), produced the highest reduction in epidermal DNA synthesis in EFAD rats, to a level almost equivalent to that in non-EFAD rats, indicating the preferential suppressive effect of barrier-enhancing substances on epidermal DNA synthesis. Since the relationship between barrier defects and increased DNA synthesis in EFAD rat skin is well established (24), our observed inhibitory effect of barrier-enhancing substances on stimulated DNA synthesis in EFAD rat skin could also be seen as merely a consequence of restoration of the barrier defect through an unknown mechanism that increases DNA synthesis. However, in this study, topical applications of pseudo-acylceramide with ester-linked linoleic acid (BRS-001) and pseudoceramide containing ether-linked linoleic acid (BRS-010) also significantly reduced cutaneous hyperplasia caused by UVB exposure or tape stripping through markedly diminishing the barrier defect. This indicated that a primary effect on the barrier function can cause a secondary reduction in hyperplasia. In contrast, linoleic acid itself, an ordinary pseudo-ceramide, and pseudo-acylceramide containing esterlinked linoleic acid with an amide-linked odd numbered fatty acid (BRS-017) (as compared with the amide-linked evennumbered fatty acid in BRS-001) did not significantly inhibit the hyperplasia. Thus, it seems likely that the potential of several pseudo-ceramides to restore the barrier defect in EFAD rat skin is well correlated with their potential to inhibit the induced epidermal hyperplasia, which, in turn, is closely associated with the increased DNA synthesis induced by exogenous stimulation. Based upon our observation that the barrier-enhancing substances tested had no substantial potential for suppressing DNA synthesis in cultured keratinocytes, our findings indicate that barrier function plays an important role in controlling epidermal DNA synthesis, as suggested in a recent report (10). Our findings that the acylceramides with ester- or ether-linked linoleic acid, but not linoleic acid itself, are effective in suppressing the induced epidermal hyperplasia through the marked improvement of barrier function in the non-EFAD state, suggest that linoleate-rich acylceramides are an intrinsic regulatory factor for epidermal barrier function despite the presence of diverse lipids in the barrier layer.

In conclusion, since this pseudo-acylceramide specifically corrected barrier defects, resulting in the reduction of epidermal hyperplasia, linoleate-rich acylceramides appear to play an important role in the mechanism of barrier function in the stratum corneum. Ongoing studies to characterize the assembly and organization of this acylceramide in the lammellar structure of stratum corneum lipids may help to clarify the role of these lipids in barrier function.

\section{Acknowledgments}

The authors wish to acknowledge the excellent technical assistance of Miss Atsuko Tsuboi.

\section{References}

1. Elias, P. M., and D. S. Friend. 1975. The permeability barrier in mammalian epidermis. J. Cell Biol. 65:180-191.

2. Wertz, P. W., E. S. Cho, and D. T. Downing. 1983. Effect of essential fatty acid deficiency on the epidermal sphingolipids of the rat. Biochim. Biophys. Acta. 753:350-355.

3. Melton, J. L., P. W. Wertz, D. C. Swartzendruber, and D. T. Downing. 1987. Effects of essential fatty acid deficiency on epidermal O-acyl-sphingolipids and transepidermal water loss in young pigs. Biochim. Biophys. Acta. 921:191197.

4. Imokawa, G., and M. Hattori. 1985. A possible function of structural lipids in the water-holding properties of the stratum corneum. J. Invest. Dermatol. 83:282-284.

5. Imokawa, G., S. Akasaki, M. Hattori, and N. Yoshizuka. 1986. Selective recovery of deranged water-holding properties by stratum corneum lipids. $J$. Invest. Dermatol. 87:758-761.

6. Imokawa, G., S. Akasaki, Y. Minematu, and M. Kawai. 1989. Importance of intercellular lipids in water-retention properties of the stratum corneum: induction and recovery study of surfactant dry skin. Arch. Dermatol. Res. 281:45-51.

7. Imokawa, G., H. Kuno, and M. Kawai. 1991. Stratum corneum lipids serve as bound-water modulator. J. Invest. Dermatol. 96:845-851.

8. Imokawa, G., S. Akasaki, O. Kuno, M. Zama, M. Kawai, Y. Minematsu, M. Hattori, N. Yoshizuka, A. Kawamata, S. Yano, and N. Takaishi. 1989. Function of lipids on human skin. J. Dis. Sci. Tec. 10:617-641.

9. Imokawa, G., S. Akasaki, A. Kawamata, S. Yano, and N. Takaishi. 1989. Water-retaining function in the stratum corneum and its recovery properties by synthetic pseudo-ceramides. J. Soc. Cosmet. Chem. 40:273-285.

10. Proksch, E., K. R. Feingold, and P. M. Elias. 1990. Barrier function regulates epidermal DNA synthesis. J. Clin. Invest. 87:1668-1673.

11. Feingold, K. R., M. Mao-Qiang, E. Proksch, G. K. Menon, B. E. Brown, and P. M. Elias. 1991. The lovastatin-treated rodent: a new model of barrier disruption and epidermal hyperplasia. J. Invest. Dermatol. 96:201-209.

12. Imokawa, G., and T. Tejima. 1989. A possible role of prostaglandins in PUVA-induced inflammation: implication by organ cutured skin. $J$. Invest. Dermatol. 92:296-300.

13. Lampe, M. A., A. L. Burlingame, J. Whitney, M. I. Williams, B. E. Brown, E. Roitman, and P. M. Elias. 1983. Human stratum corneum lipids: characterization and regional variations. J. Lipid Res. 24:120-130.

14. Elias, P. M. 1983. Epidermal lipids, barrier function, and desquamation. J. Invest. Dermatol. 80:44-49. 
15. Grubauer, G., K. R. Feingold, R. M. Harris, and P. M. Elias. 1989. Lipid content and lipid type as determinants of the epidermal permeability barrier. $J$. Lipid Res. 30:89-96.

16. Elias, P. M., B. E. Brown, and V. A. Ziboh. 1980. The permeability barrier in essential fatty acid deficiency: evidence for a direct role for linoleic acid in barrier function. J. Invest. Dermatol. 74:230-239.

17. Wertz, P. W., and D. T. Downing. 1983. Ceramides of pig epidermis: structure determination. J. Lipid Res. 24:759-765.

18. Nugteren, D. H., E. Christ-Hazelhof, A. van der Beek, and U. M. T Houtsmuller. 1985. Metabolism of linoleic acid and other essential fatty acids in the epidermis of the rat. Biochim. Biophys. Acta. 834:429-436.

19. Elliott, W. J., H. Sprecher, and P. Needleman. 1985. Physiologic effects of columbinic acid and its metabolites on rat skin. Biochim. Biophys. Acta. 835:158-160.

20. Elliott, W. J., A. R. Morrison, H. Sprecher, and P. Needleman. 1985.
The metabolic transformation of columbinic acid and the effect of topical application of the major metabolites on rat skin. J. Biol. Chem. 260:987992.

21. Bowser, P. A., D. H. Nugteren, R. J. White, U. M. T. Houtsmuller, and C. Prottey. 1985. Identification, isolation and characterization of epidermal lipids containing linoleic acid. Biochim. Biophys. Acta. 834:419-428.

22. Grubauer, G., P. M. Elias, and K. R. Feingold. 1989. Transepidermal water loss: the signal for recovery of barrier structure and function. J. Lipid Res. 30:323-333.

23. Wood, L. C., S. M. Jackson, P. M. Elias, C. Grunfeld, and K. R. Feingold. 1992. Cutaneous barrier perturbation stimulates cytokine production in the epidermis of mice. J. Clin. Invest. 90:482-487.

24. Lowe, N. J., and R. B. Staughton. 1977. Essential fatty acid deficient hairless mouse: a model of chronic epidermal hyperproliferation. Br. J. Dermatol. 96:155-162. 